\title{
Distal Ischemia of the Four Limbs: A Case Report and Literature Review
}

\author{
Hector Masaragian ${ }^{1}$, Leonel Rega ${ }^{2}$, Nicolas Ameriso $^{3}$, Fernando Perin ${ }^{4}$, Hernan Coria ${ }^{5}$, Luciano Mizdraji ${ }^{6}$, Sabrina Cicarella $^{7}$
}

\begin{abstract}
The symmetrical peripheral gangrene syndrome is characterized by ischemic distal lesions in two or more extremities, without the obstruction of large vessels. The ischemia affecting extremities takes place when the arterial blood flow is insufficient to satisfy the metabolic demands of muscles in rest. We present a 22-year-old patient treated with norepinephrine after a septic shock suspicion who presented distal ischemia of fingers, toes, and scalp. There is no consensus about the initial treatment. Improved data gathering is necessary for a better understanding of the mechanisms involved, which could improve treatment and prevent amputation as the final therapeutic option.

Keywords: Bone infarction, Distal ischemia, Norepinephrine, Sepsis, Symmetrical peripheral gangrene.

Journal of Foot and Ankle Surgery (Asia Pacific) (2019): 10.5005/jp-journals-10040-1107
\end{abstract}

\section{INTRODUCTION}

The symmetrical peripheral gangrene syndrome is characterized by ischemic distal lesions in two or more extremities, without the obstruction of large vessels.

This condition was first reported by Jonathan Hutchinson in $1891 .^{1}$ He described a patient who presented distal finger necrosis in all extremities. Since then, it has been clear that this syndrome is associated with low cardiac output and several other medical conditions such as intravascular disseminated coagulation (IDC), ${ }^{2}$ sepsis, ${ }^{3}$ low body temperature, and vasoactive drugs administration. ${ }^{4}$

The ischemia affecting extremities takes place when the arterial blood flow is insufficient to satisfy the metabolic demands of muscles in rest. This is seen in 1-2\% of patients older than 50 years with a peripheral arterial disease (PAD).

Noradrenalin establishes a hemodynamic state and increases perfusion to the principal organs, affecting alpha-1 receptors. On some occasions, this mechanism may cause ischemic distal necrosis due to the decreased perfusion in terminal organs.

\section{Case Description}

We treated a 22-year-old male who was received in the emergency service with vomits, diarrhea (seven depositions), and abdominal pain, which did not improve with medication or antalgic posture, and he had fever 24 hours after a surgical rhinoplasty procedure. Hospitalization was decided, but 48 hours later, his condition evolved with hypotension (70/40 $\mathrm{mm} \mathrm{Hg}$ ), tachypnea, and bad peripheral blood perfusion. He received $1,500 \mathrm{~mL}$ of crystalloids and a laboratory test was made hematocrit $48 \%$, hemoglobin $16 \mathrm{~g} / \mathrm{dL}$, urea $146 \mathrm{mg} / \mathrm{dL}$, creatinine $4.65 \mathrm{mg} / \mathrm{dL}$, and lactic acid $9.5 \mathrm{mmol} / \mathrm{L}$. The acid-base balance presented arterial blood PH of 7.29, $\mathrm{PaO}_{2} 81$ $\mathrm{mm} \mathrm{Hg}, \mathrm{HCO}_{3} 10 \mathrm{mEq} / \mathrm{L}, \mathrm{PaCO}_{2} 22 \mathrm{~mm} \mathrm{Hg}$, base excess $-13 \mathrm{mEq}$, and $\mathrm{SaO}_{2} 93 \%$. Under the suspicion of septic shock after surgical procedure, the patient referred to the intensive care unit.

Due to a poor pulmonary mechanic condition, respiratory support was needed. Empiric treatment consisted of noradrenalin (1.5 $\mu \mathrm{g} / \mathrm{kg} / \mathrm{minute})$ and blood cultures prior to antibiotic therapy with meropenem, daptomycin, clindamycin, and immunoglobulin administration. Severe systolic function alteration
${ }^{1-7}$ Department of Foot and Ankle, Dr Masaragian-Cirugiadelpie.net, Autonomous City of Buenos Aires, Argentina

Corresponding Author: Hector Masaragian, Department of Foot and Ankle, Dr Masaragian-Cirugiadelpie.net, Autonomous City of Buenos Aires, Argentina, Phone: +54 1152494685, e-mail: masa@cirugiadelpie. net

How to cite this article: Masaragian $\mathrm{H}$, Rega $\mathrm{L}$, Ameriso $\mathrm{N}$, et al. Distal Ischemia of the Four Limbs: A Case Report and Literature Review. J Foot Ankle Surg (Asia Pacific) 2019;6(2):46-51.

Source of support: Nil

Conflict of interest: None

was experienced and determined by an echocardiogram test after 48 hours. Dobutamine infusion with a dose of $5 \mu \mathrm{g} / \mathrm{kg} /$ minute for 72 hours was indicated. Noradrenaline dose was decreased to $0.03 \mu \mathrm{g} / \mathrm{kg} / \mathrm{minute}$ with an echocardiogram control showing good heart motility, no ventricle dilatation with posterior hypertrophy, and normal systolic function. IDC was suspected, and 1,500 IU/day of heparin was infused, decreasing to $10,000 \mathrm{IU} /$ day the following 24 hours and suspending treatment after 72 . Since good clinical evolution was observed, respiratory support was removed.

Seventy-two hours after symptoms started, distal cyanosis of the four limbs presented (Figs 1 and 2), and scalp lesions were observed (Fig. 3). At this time, the only therapeutic measure available was expectant management until reperfusion of distal tissues. Lesions delimitation was seen a week after initial symptoms began (Figs 4 and 5).

Daily wound dressing changes, and plastic surgeon evaluation continued for the next 10 days when left hallux necrosis was accepted irreversible due to low tissue perfusion (Fig. 6), deciding a distal phalanx amputation. During the procedure, distal bone necrosis was observed. On the right hallux, the only resection of superficial necrotic areas and the nail matrix was necessary (Fig. 7).

Satisfactory wound healing with complete reepithelization of necrotic areas was seen (Fig. 8).

Ankle magnetic resonance imaging of the right foot and ankle were obtained, with bone infraction of the distal tibia, talus, 

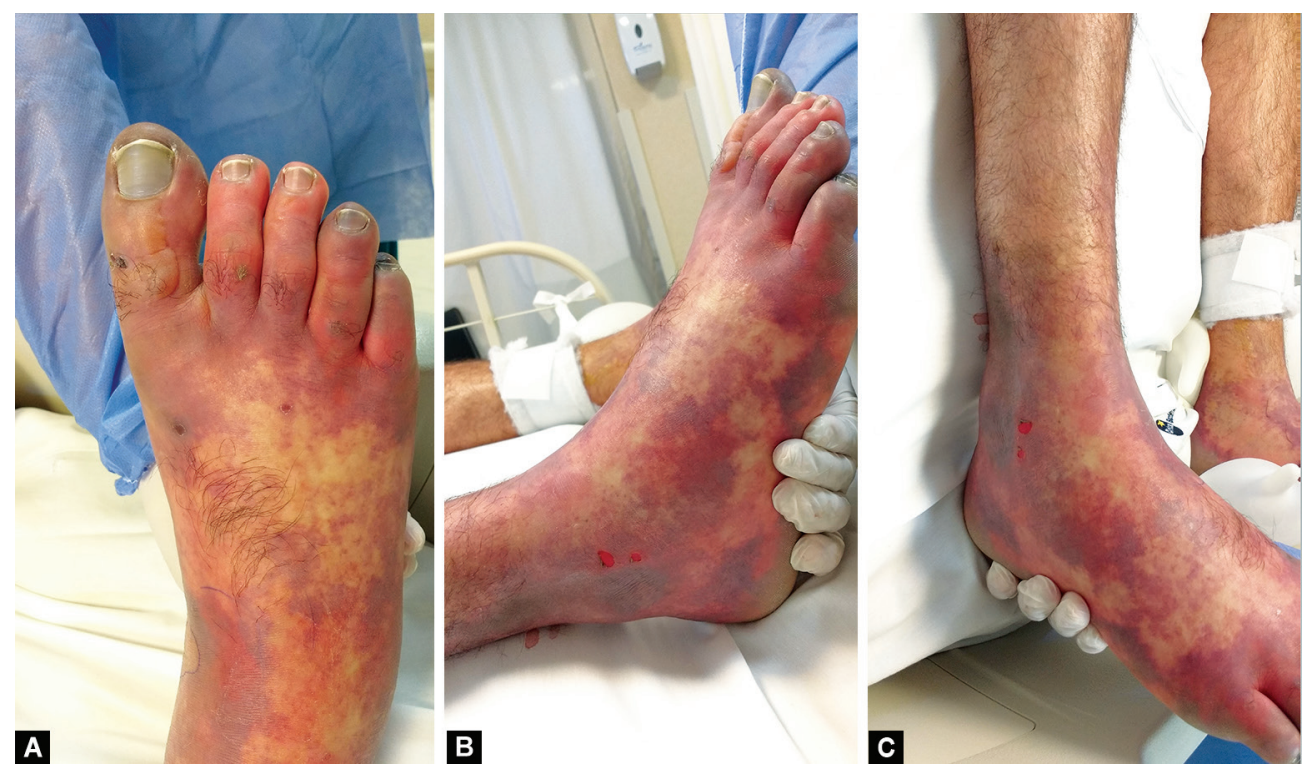

Figs $1 \mathrm{~A}$ to C: Right foot at 72 hours' posterior to initial symptoms

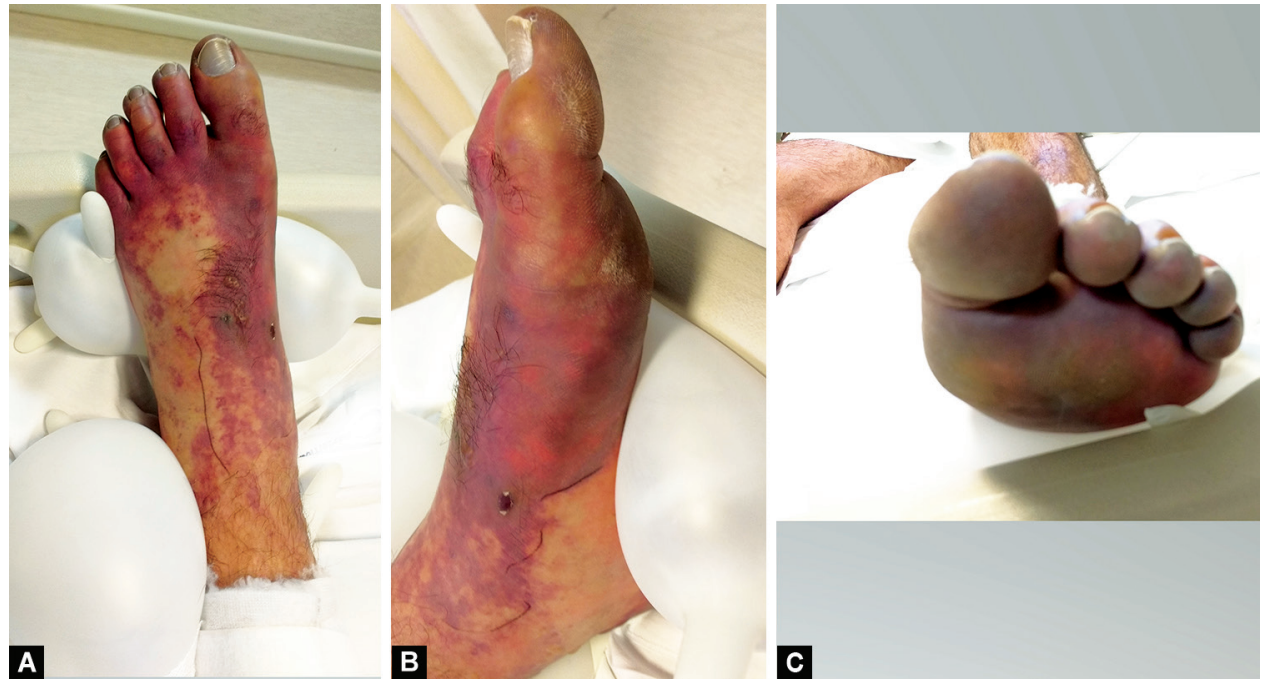

Figs $2 \mathrm{~A}$ to $\mathrm{C}$ : Left foot at 72 hours' posterior to initial symptoms
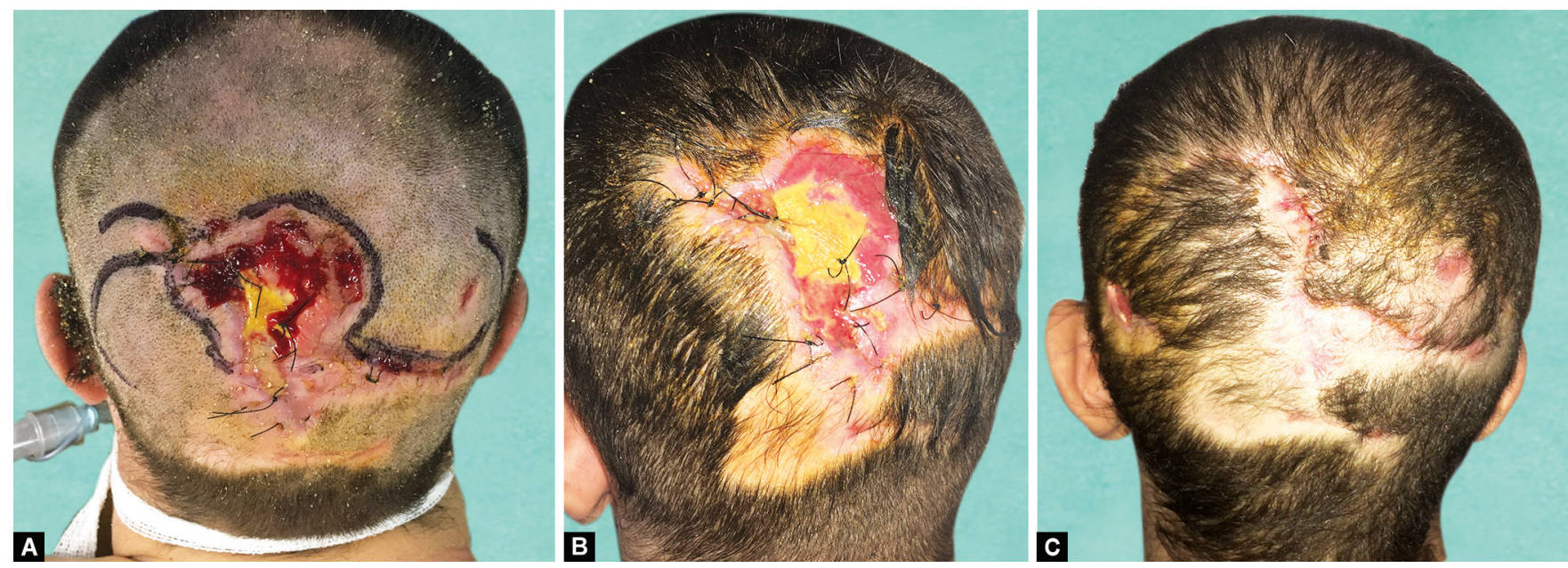

Figs 3 A to C: Scalp lesion and its evolution 
calcaneus, navicular, cuboid, cuneiforms, and metatarsal bases (Fig. 9).

The final clinical diagnosis determined was toxic septic shock due to Staphylococcus aureus. The patient was discharged after 34 days.

\section{Discussion}

It has been accepted that the symmetrical peripheral gangrene syndrome is a complication of low cardiac output. In 1938 Fishberg suggested that this condition was a result of low peripheral perfusion due to the excessive production of vasoconstrictors. ${ }^{5}$ This theory is supported by histopathological studies, which showed small vessel constriction when intraluminal pressure drops below the critical value of $36-60 \mathrm{~mm} \mathrm{Hg} ., 7$

Several conditions may induce the development of this pathology, including sepsis (bacterial, viral and parasitic),

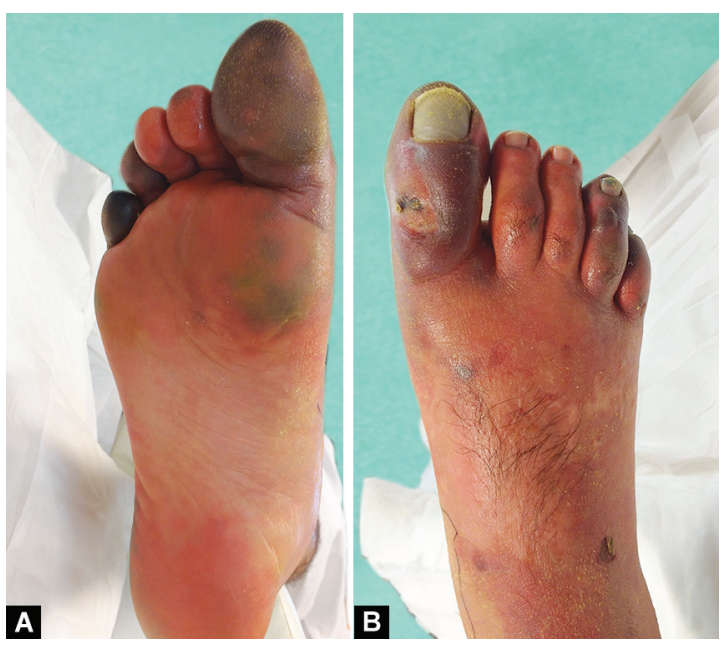

Figs 4A and B: Right foot after a week: (A) Plantar view; (B) Dorsal view

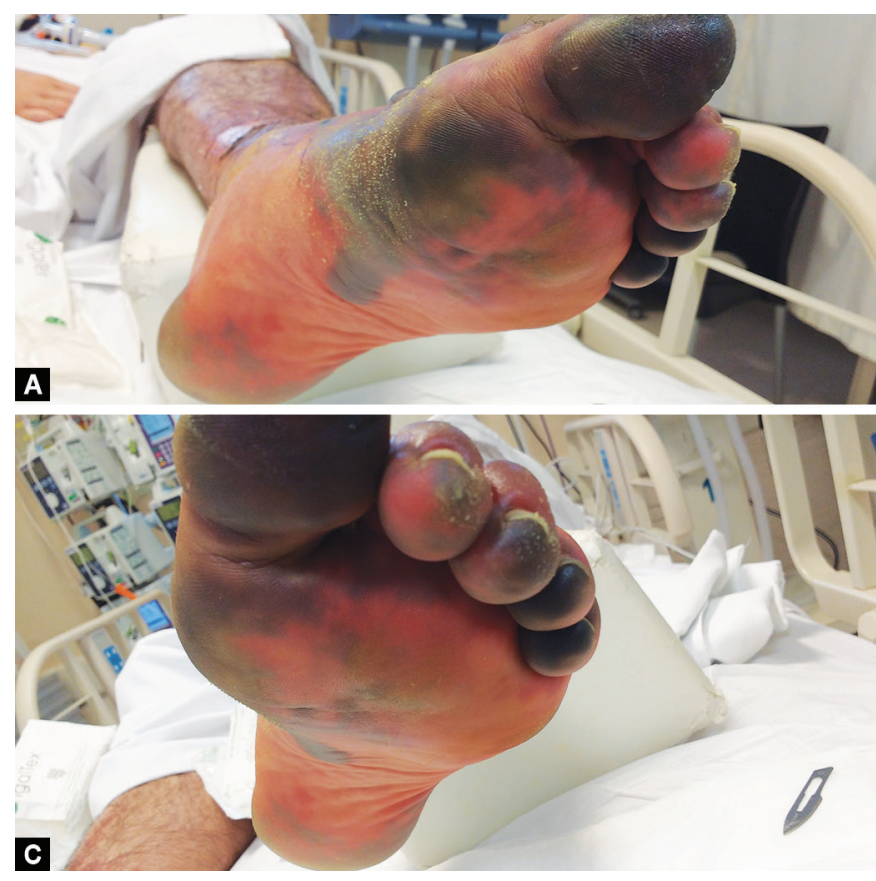

Figs 5A to D: Left foot after a week cardiovascular (cardiac failure, hypovolemic shock, pulmonary embolism, supraventricular tachycardia), drugs, neoplastic conditions, and others.

Peripheral gangrene has also been associated with vasoactive drugs such as vasopressin ${ }^{8,9}$ and dopamine. ${ }^{4}$ It is likely that the vasospasm effect of noradrenaline, which is more intense in the distal vascular supply, produces the same pathology. In our patient, we suspect that the noradrenaline infusion was the underlying cause.

In septic shock, cardiac output drops and the vascular system increases its resistance. This is due to the efforts of the body to maintain adequate perfusion to the heart and brain, while blood flow to the peripheral organs drops. Since this condition has a high mortality rate, the use of excessive vasoconstriction drugs is used to stabilize the patient. Noradrenaline is the preferred treatment to maintain hemodynamic support when dopamine has failed. It induces an increase of contractility and vasoconstriction after affecting $a$ and $\beta$-adrenergic receptors.

The treatment for symmetrical peripheral gangrene syndrome is usually unsatisfactory. Damage control is the aim. Several therapies, medication, and maneuvers have been proposed, but there is no proof they could prevent progression or reverse the distal necrosis. In 2011 Ghosh and Bandyopadhyay described three treatment groups: general, medical, and surgical. ${ }^{10}$ General treatment consists in minimizing vasopressors and adding padding and vascular boots. Medical treatment includes intravenous fluids, parenteral antibiotics, anticoagulation, intravenous sodium nitroprusside, papaverine, streptokinase, dextran, hyperbaric oxygen, sympathetic blockers, intravenous prostaglandins (epoprostenol), topical nitroglycerine, and plasmapheresis. Finally, surgical treatment is indicated once lesions are delimitated. Amputation of irreversible areas is indicated. In the areas where amputation is not an option, skin grafting is carried out. Due to the rarity of this entity, there is no clear treatment protocol to this date.

In a recent study, Levy et al. ${ }^{11}$ suggest the distal necrosis may not be due to the vasopressor therapy, but a consequence
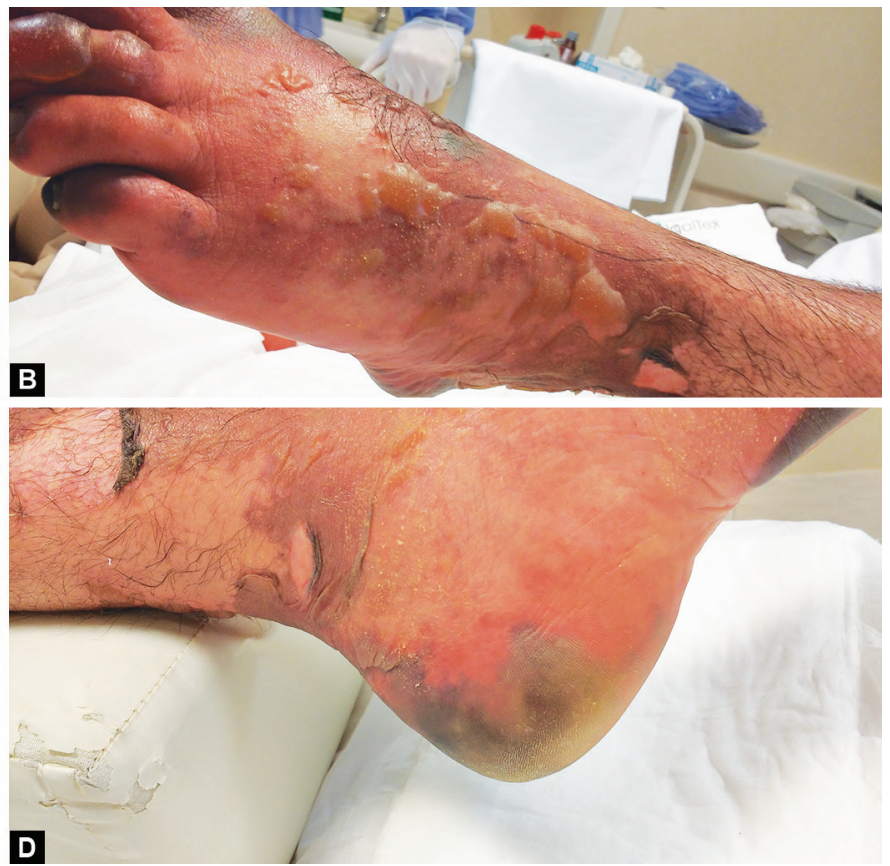

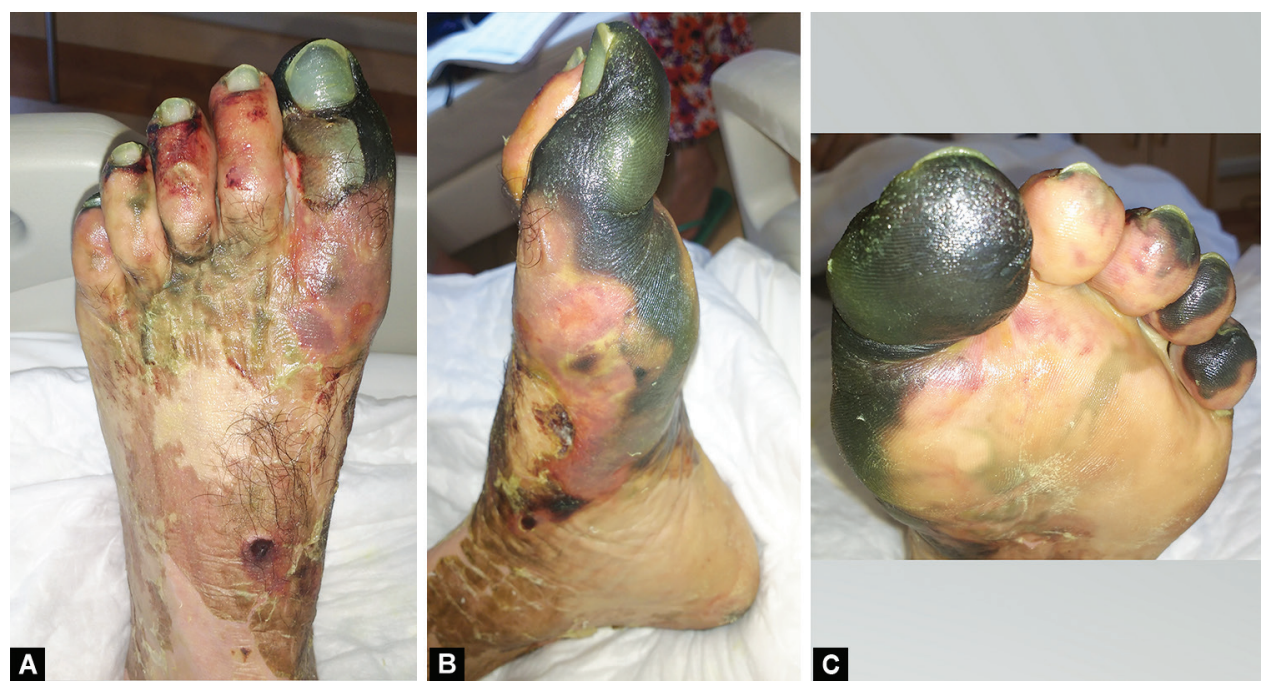

Figs 6 A to $C$ : Left foot at 10 days
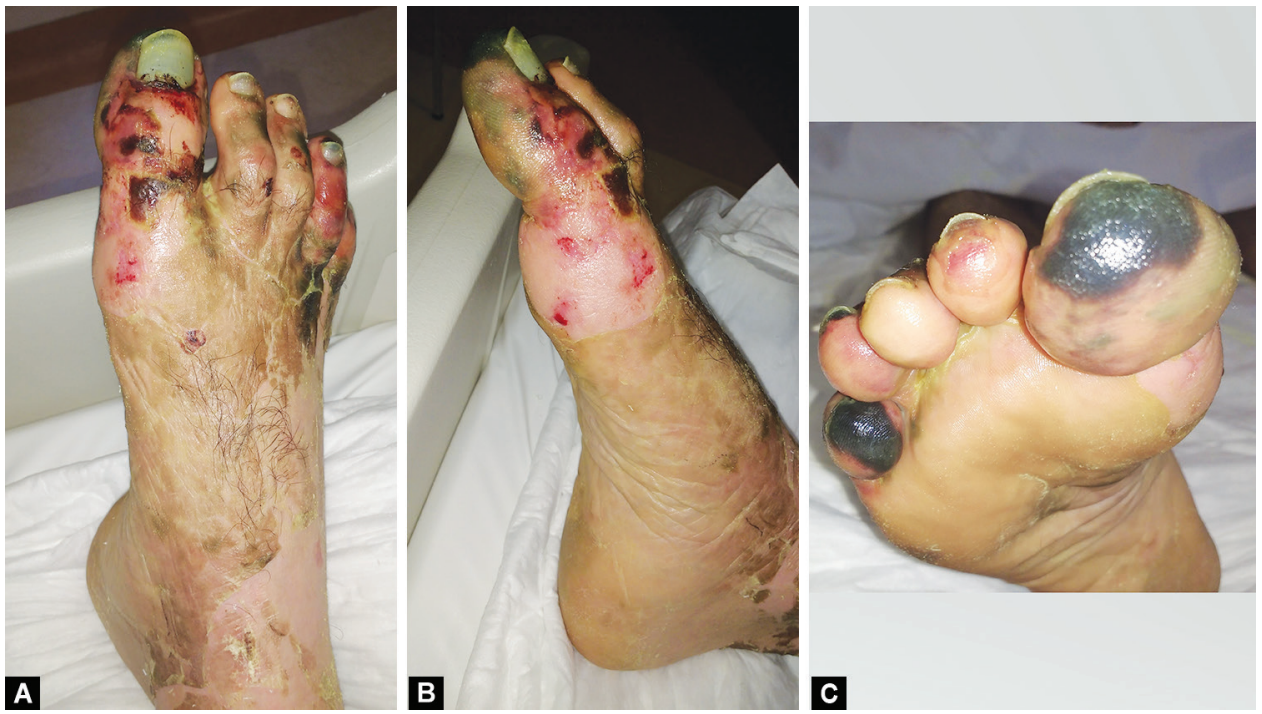

Figs 7A to C: Right foot at 10 days

of disseminated intravascular coagulopathy (DIC) and/or shock liver, a complication which our patient presented. They conducted a systematic review of high dose vasopressors treatment and found no direct link of distal ischemia to the therapy. On the contrary, DIC incidence or other complications such as shock liver was not informed in the studies included in the review. Since DIC is present in over $35 \%$ of septic shock patients, the necrosis could be considered as a result of thrombotic occlusion of the microvasculature. They also refer to a typical median timeframe of 3 days in which ischemic lesions become evident, bearing in mind that if vasopressors were the cause, they should be seen immediately.

In 2018 four case report studies were presented. Pacori et al. presented a case report of a 39-year-old patient who required 5 days of a high dose of vasopressors due to a septic shock secondary to a vaginal ring infection, but no laboratory details or DIC were reported. The only treatment described was amputation.

Albano et al. ${ }^{12}$ describe another case report of a 45 -year-old patient with septic shock, in this case, due to a urinary tract infection.
DIC with thrombocytopenia and prolonged coagulation times were observed. Treatment consisted of isosorbide dinitrate, which was substituted for prostaglandin 12, but with neither clinical improvement was seen. Once again, amputation was required as a final treatment.

Jung et al. reported four cases, all of them related to septic shock. ${ }^{13}$ Once again, no references to DIC or hepatic failure presence were made, and amputation was necessary.

Tan et al. described a case of distal ischemia in a 42-yearold male after collision with his motorbike. ${ }^{14}$ He presented right frontal intraparenchymal bleeding, open wound right femur fracture, right fracture, and multiple rib fractures. His critical state demanded a high dose of vasopressors, but they diagnosed DIC and hepatic failure, with documented thromboembolism, which they considered responsible for the distal necrosis.

Finally, in 2019, Vidal et al. presented a case report of a 14-yearold male who suffered distal ischemia of the four limbs due to a Staphylococcus aureus septic shock. ${ }^{15}$ In this case, the authors 

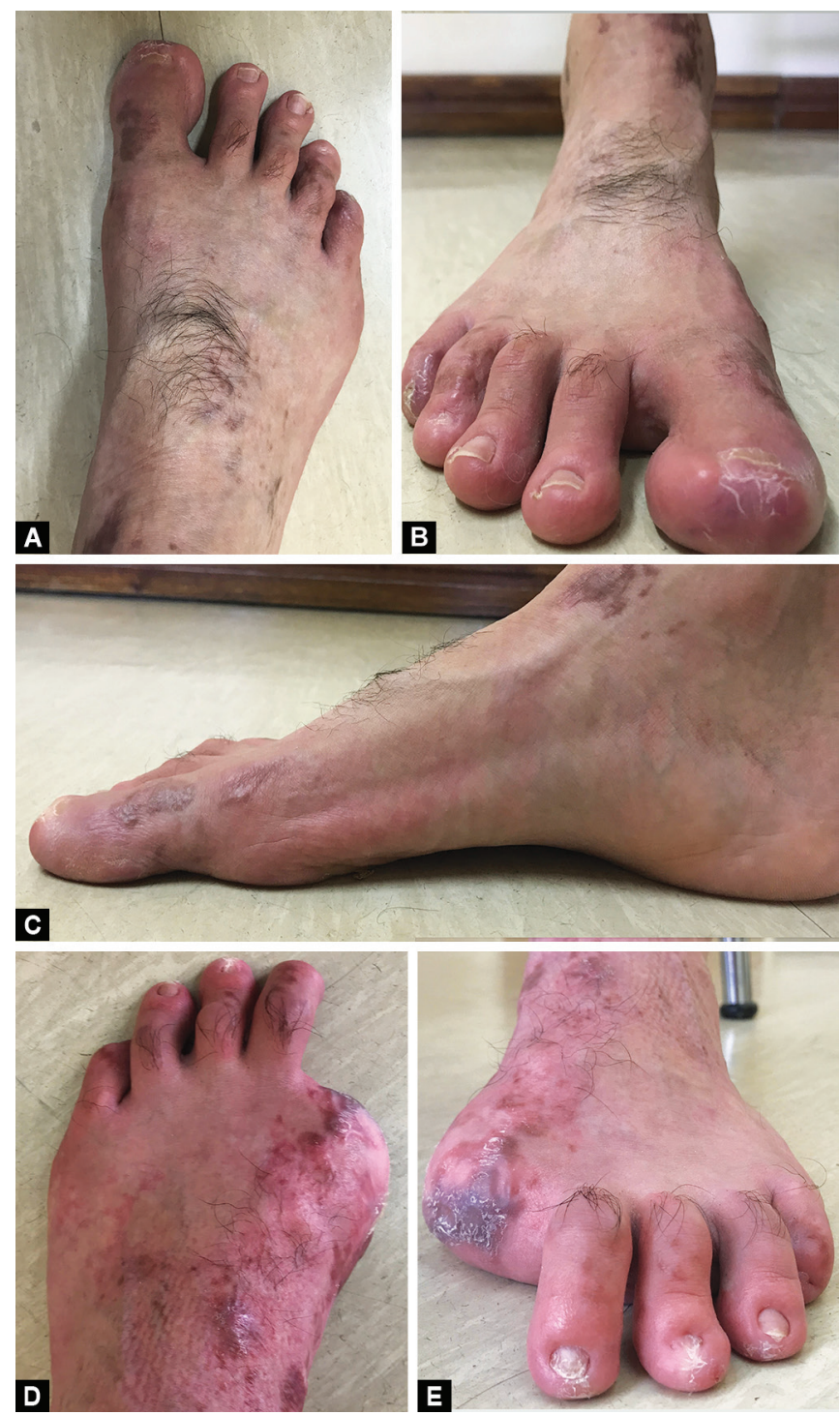

Figs $8 \mathrm{~A}$ to $\mathrm{E}$ : Both feet 6 months after treatment: top and middle images right foot; bottom images left foot

analyzed the amputated regions with a light microscope observing medium-sized vessels with perivascular infiltrates, endothelium destruction, and thrombosis. No microvascular lesions were seen. They attribute the thrombosis to the vascular lesions due to pathogen virulence (an extensive study is presented) and the coagulation balance disorders.

\section{Conclusion}

Although more case reports of this unusual pathology seem to be reported every year, there is still no consensus about its cause, and therefore its treatment. What initially seemed to be an obvious cause of high vasopressor dosage is now suspected to be multifactorial. After the literature review, new mechanisms, including disseminated vascular coagulopathy, hepatic failure, and bacterial vascular lesion appear, as plausible causes for the final lesions. A common conclusion to all studies is that prospective research with improved data gathering is necessary for a better understanding of the mechanisms involved, which could improve
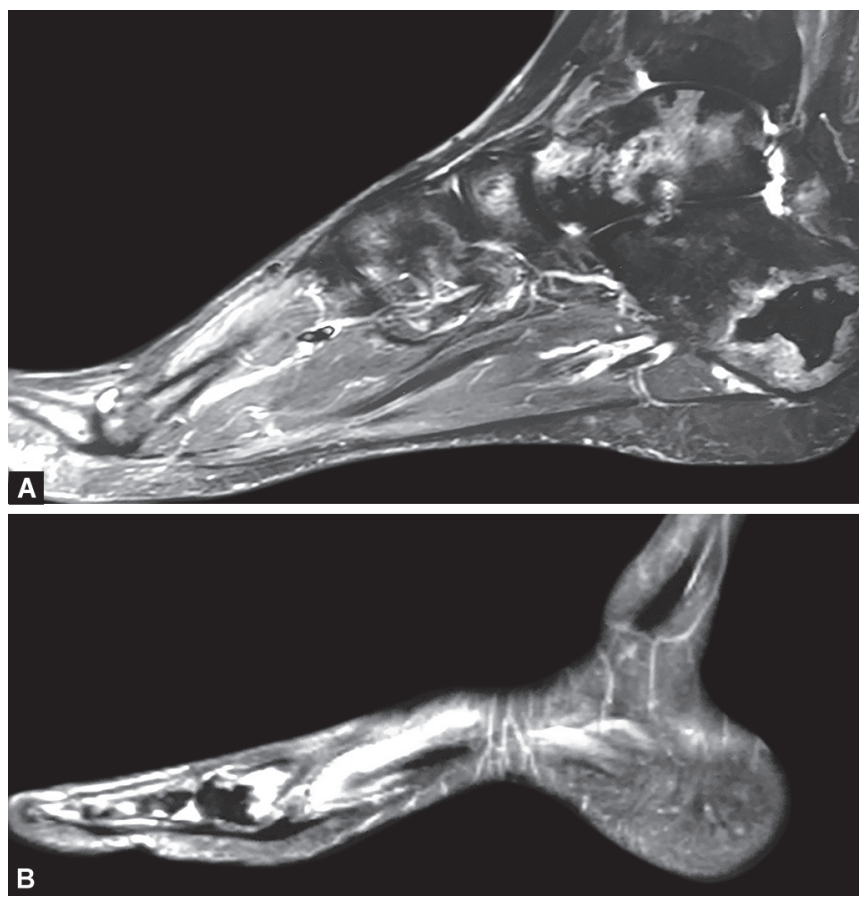

Figs $9 \mathrm{~A}$ and $\mathrm{B}$ : Magnetic resonance imaging of the right foot and ankle: bone infraction of distal tibia, talus, calcaneus, navicular, cuboid, cuneiforms and metatarsal bases

the initial treatment and prevent amputation as the final therapeutic option. Unfortunately, we can't provide alternative conclusion, motivating further multivariable studies in patients with distal ischemia, including hepatic function valorization, coagulation status, microvascular, and macrovascular thrombosis evaluation and pathogen recording as to improve the current treatment alternatives.

\section{References}

1. Hutchinson J. Notes of uncommon cases. Br Med J 1891;2(1592):8-9. DOI: $10.1136 / \mathrm{bmj} .2 .1592 .8$.

2. Molos MA, Hall JC. Symmetrical peripheral gangrene and disseminated intravascular coagulation. Arch Dermatol 1985;121(8):1057-1061. DOI: 10.1001/archderm.1985.01660080111027.

3. Johansen K, Murphy T, Pavlin E, et al. Digital ischemia complicating pneumococcal sepsis: reversal with sympathetic blockade. Crit Care Med 1991;19(1):114-116. DOI: 10.1097/00003246-19910100000025.

4. Winkler MJ, Trunkey DD. Dopamine gangrene: association with disseminated intravascular coagulation. Am J Surg 1981;142(5): 588-591. DOI: 10.1016/0002-9610(81)90432-3.

5. Hayes MA, Yau EHS, Hinds CJ, et al. Symmetrical peripheral gangrene: association with noradrenaline administration. Intensive Care Med 1992;18(7):433-436. DOI: 10.1007/BF01694349.

6. Simman R, Phavixay L. Bilateral toe necrosis resulting from norepinephrine bitartrate usage. Adv Skin Wound Care 2013; 26(6):254-256. DOI: 10.1097/01.ASW.0000431083.77517.fd.

7. Shin JY, Roh S, Lee N, et al. Ischemic necrosis of upper lip, and all fingers and toes after norepinephrine use. J Craniofac Surg 2016;27(2):453-454. DOI: 10.1097/SCS.0000000000002463.

8. Forrest $\mathrm{CR}$, Pang $\mathrm{CY}$, Zhong AG, et al. Role of noradrenaline in the pathogenesis of skin flap ischemic necrosis in the pig. J Surg Res 1990;48(3):237-244. DOI: 10.1016/0022-4804(90)90219-R.

9. Kim EH, Lee SH, Byun SW, et al. Skin necrosis after a low-dose vasopressin infusion through a central venous catheter for treating 
septic shock. Korean J Intern Med 2006;21(4):287-290. DOI: 10.3904/ kjim.2006.21.4.287.

10. Ghosh SK, Bandyopadhyay D. Symmetrical peripheral gangrene. Indian J Dermatol Venereol Leprol 2011;77(2):244-248. DOI: 10.4103/0378-6323.77481.

11. Levy JH, Ghadimi K, Faraoni D, et al. Ischemic limb necrosis in septic shock: what is the role of high-dose vasopressor therapy? J Thromb Haemost 2019;17(11):1973-1978. DOI: 10.1111/jth.14566.

12. Albano MN, Brazão SG, Caroço TV, et al. Rare case of symmetrical peripheral gangrene due to septic shock, disseminated intravascular coagulation and inotropic use. Ann Med Surg (Lond) 2018;35:103-107. DOI: 10.1016/j.amsu.2018.09.025.
13. Jung KJ, Nho JH, Cho HK, et al. Amputation of multiple limbs caused by use of inotropics. Medicine (Baltimore) 2018;97(5):e9800. DOI: 10.1097/MD.0000000000009800.

14. Tan JH, Mohamad Y, Tan CLH, et al. Concurrence of symmetrical peripheral gangrene and venous limb gangrene following polytrauma: a case report. J Med Case Rep 2018;12(1):131. DOI: 10.1186/ s13256-018-1684-1.

15. Vidal C, Moulin F, Nassif $X$, et al. Fulminant arterial vasculitis as an unusual complication of disseminated staphylococcal disease due to the emerging CC1 methicillin-susceptible Staphylococcus aureus clone: a case report. BMC Infect Dis 2019;19(1):302. DOI: 10.1186/ s12879-019-3933-3. 